\title{
A Simplified QoS scheme using Virtual Link in MPLS based backbone network
}

\author{
ChulWoo Kim, Seung-Joon Seok, Jung-Dae Kim, and Chul-Hee Kang \\ Dep. of Electronics Engineering Korea University
}

\begin{abstract}
In this paper, a simplified QoS scheme is proposed. The proposed scheme uses a Virtual Link which is a Label Switched Path (LSP) set connected from Ingress Label Egde router (LER) to Egress LER. LSPs of the virtual link must be managed dynamically according to network status. The traffic in the virtual link is distributed among LSPs to reduce service unbalancing by the flow. In this proposed model, Per-Hop-Behavior (PHB) is implemented in each LSP of ingress LER and Core LSRs just guarantee bandwidth. These features make it simple and easy to provide Quality of Service in MPLS network. The characteristics of the proposed scheme and the IETF scheme are compared logically and analyzed through simulations.
\end{abstract}

Key words: MPLS, QoS, Virtual Link

\section{INTRODUCTION}

In the past several years, works on QoS enabled network, such as Differentiated Service (DiffServ), to provide growing multi-class services and MultiProtocol Label Switching(MPLS) to improve the forwarding speed of routers led to several propositions in the IETF [1][2]. So far, however, these works have been considered independently for their own purposes. Recently IETF has presented supporting Quality of Service using DiffServ over MPLS network [3][4].

There are some ways of supporting QoS on a MPLS based backbone network. Two of them are using Traffic Engineering with explicit routing and DiffServ over MPLS. DiffServ over MPLS classifies packets into a small number of aggregated flows or service classes only at an Ingress LER and applies PHB to flow aggregates that have been appropriately marked at every router as a standard DiffServ [3]. That is an integrated model of DiffServ and MPLS network. To provide DiffServ in MPLS, Differentiated Services Code Point (DSCP) in IP header should be classified into MPLS labels. The Figure 1 shows this conventional model.

Currently, two label encoding schemes are proposed, which are E-LSP and L-LSP 
mechanisms [2][5]. E-LSPs transport multiple Ordered Aggregates (OAs), so that the EXP field of the MPLS Shim Header conveys to the Label-Switch Router(LSR) the PHB to be applied to the packet including both information about the packet's scheduling treatment and its drop precedence. However, it carries up to only eight behavior aggregates (BAs) on a LSP for a given Forward Equivalence Class (FEC). So, when more traffic needs to be accommodated, load sharing is required. On the other hand, L-LSPs only transport a single OA, so that the packet scheduling treatment is inferred by the LSR exclusively from the packet's label value while the packet's drop precedence is conveyed in the EXP field of the MPLS Shim Header. It uses separate labels for each class. Therefore, it introduces more signaling operations, label management complexity and increases the total number of labels.

The architecture of backbone network should be as simple as possible to transport huge traffic at high speed. In fact, originally MPLS was developed to improve packet forwarding performance in back bone routers by swapping short, fixed length identifiers ('labels'), instead of cumbersome look-ups and routing in each and every case. However, the above mechanism can make the network processing complexed because every MPLS router must apply PHB. In this paper, a simplified QoS scheme using virtual link with some modifications only for LERs is proposed. The next section describes the concept of the proposed model using the virtual link and an algorithm to manage the virtual link and section 3 explains strong points and weak points through the logical comparisons between the IETF scheme and the proposed scheme. In section 4 , our proposed scheme is evaluated through several simulations and finally section 5 concludes this paper.

\section{A SIMPLIFIED QOS SCHEME USING VIRTUAL LINK IN MPLS NETWORK}

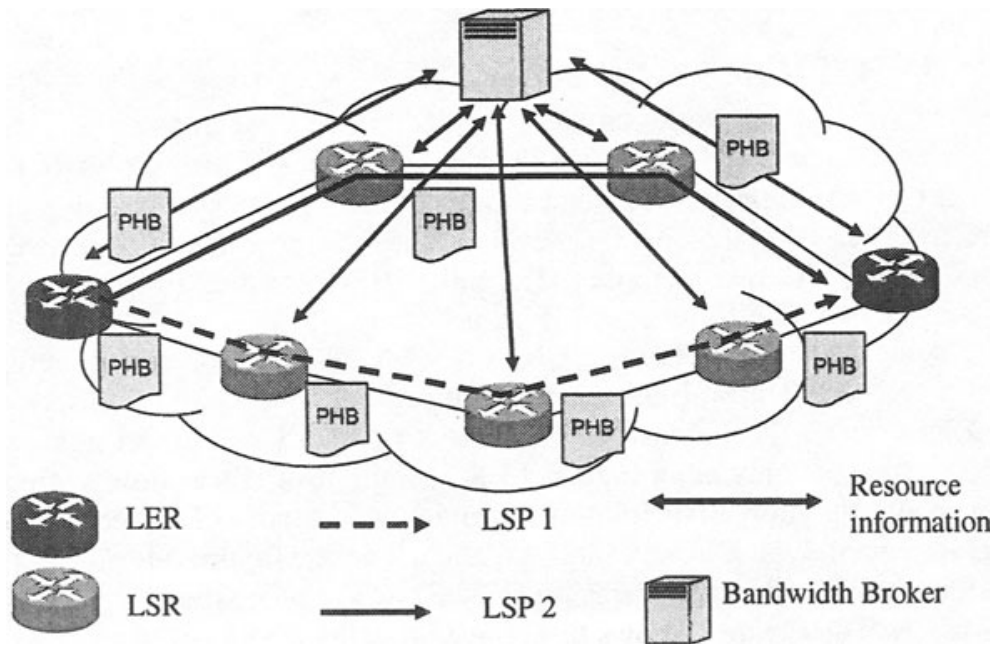

Figure 1. The conventional model proposed by IETF 


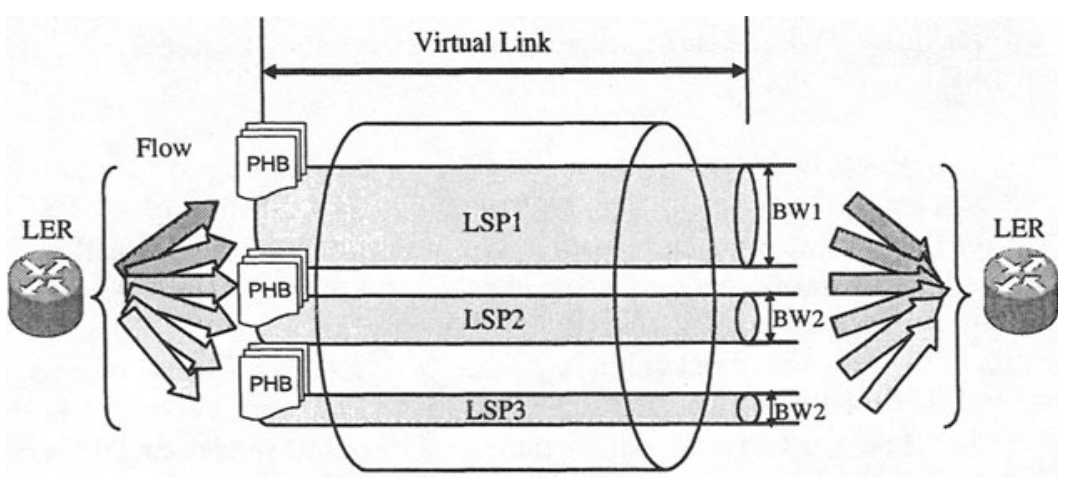

Figure 2. Virtual Link

In this section, we define virtual Link concept and describe a mechanism to adopt this concept in a legacy MPLS network.

\subsection{Virtual Link Concept}

In this paper, a virtual link is defined as a set of LSP between ingress LER and egress LER. Ingress LER assigns a PHB function to each LSP of the virtual link, as if its next node is egress LER. Also, A LSP guarantees reserved bandwidth. It can be allocated a certain bandwidth using control protocol such as CR-LDP or RSVP-TE whenever it is required. Figure 2 illustrates virtual link. In this scheme, Label Switch Routers (LSRs), that are core routers forwarding packets using label swapping, carry out single service level scheduling, not multiple service level complex scheduling performed in DiffServ. This is same as that in current MPLS network. Thus, only boundary edge nodes need to be extended to support QoS in this scheme.

\subsection{The Network Environments}

Before describing a mechanism for applying our scheme, we first assume several network environments as follows.

(1) The flow request includes information of the service class and required bandwidth.

(2) There can be one more LSPs between the same ingress LER and egress LER. Also, they might have different characteristics in packet delay, bandwidth and etc.

(3) The resource of LSPs in the virtual link can be managed dynamically.

(4) LSPs for the virtual link are set up by CR-LDP or RSVP-TE and the LSPs guarantee their reserved bandwidth. 
(5) Each LSP of the virtual link is allocated a PHB functional module. Although several flows have the same service class, each flow is treated by the PHB of its LSP.

If a request arrives and there is an existing LSP corresponding to the request, the request is accepted in the existing LSP by extending the bandwidth of the LSP or in new LSP for the request. This can improve link utilization and reduce call blocking probability. Also, we propose a QoS agent at each ingress node to manage the virtual link. In the proposed model, every node exchanges and manages network information and reserves bandwidth, and the QoS agent at every ingress node determines LSPs for the virtual link and manages it. The proposed model is shown in Figure 3. While the conventional model is a integrated model of DiffServ and MPLS, the proposed model can be considered as a overlay model of a logical QoS network and MPLS network.

\subsection{Conceptual Method for virtual link management}

Thus, there are two problems in virtual link management when the virtual link concept is used. One is what LSPs should be selected for the virtual link between an ingress LER and a egress LER and the other is how all flows are assigned to the LSPs. A conceptual method to solve these problems is as follows.

(1) One flow must be forwarded in only one LSP.

(2) A set of all paths from ingress to egress is defined as $\left\{\mathrm{P}_{k}(i, j): \operatorname{PL}\left(\mathrm{P}_{\mathrm{k}}(i, j)\right)<\right.$ max_hop_count \}. The max_hop_count can be the maximum length of LSP included in the virtual link.

- $\mathrm{P}_{\mathrm{k}}(i, j)$ denotes $\mathrm{k}^{\text {th }}$ path from ingress $i$ from egress $j$

- $\operatorname{PL}\left(\mathrm{P}_{\mathrm{k}}(i, j)\right)$ denotes Path length of $\mathrm{P}_{\mathrm{k}}(i, j)$

(3) The virtual link from ingress $i$ to egress $j$ is determined by $\operatorname{Max}\left(\left\{\operatorname{PB}\left(\mathrm{P}_{k}(i, j)\right)\right.\right.$ : $\mathrm{PL}\left(\mathrm{P}_{\mathrm{i}}(i, j)\right)<\max \_$hop_count $\left.\}\right)$

- $\mathrm{PB}\left(\mathrm{P}_{\mathrm{k}}(i, j)\right)$ : Minimum unused bandwidth through $\mathrm{k}^{\text {th }}$ path from ingress $\mathrm{i}$ to egress $\mathrm{j}$.

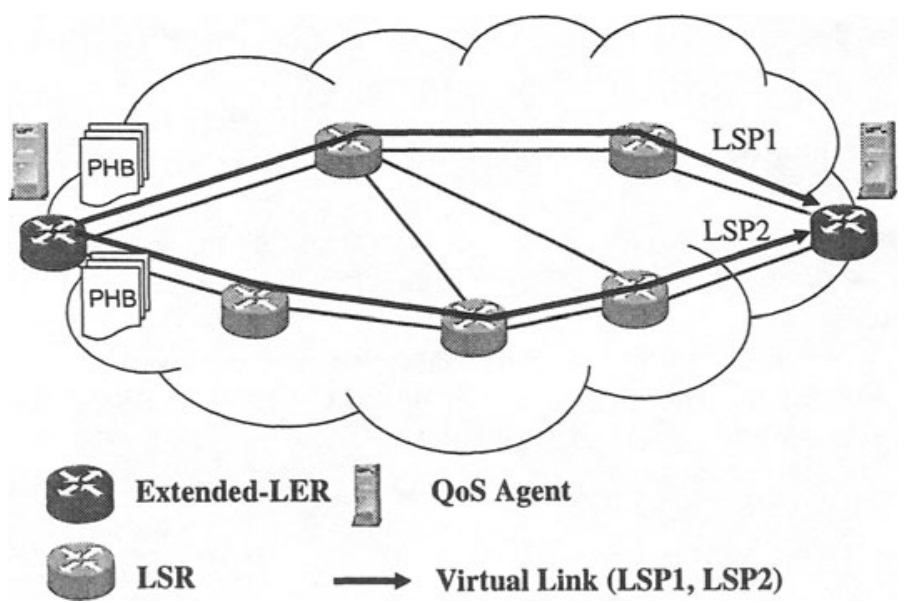

Figure 3. The Proposed model 
(4) The number of LSPs in the virtual link must not exceed threshold count, $\mathrm{K}$.

(5) The traffic is distributed among LSPs by the flow.

(6) If there are several LSPs in the virtual link, the proportion of the service classes in the all LSPs included in the virtual link should be similar.

(7) When a flow is terminated, the LSP's resource must be reduced. If there is no flow in the LSP, this will be terminated. If the service classes are unbalanced, the LSP can be merged into other LSP of the same virtual link, or the service classes can be redistributed.

(8) In the redistribution, existing flow distribution among LSPs is deviated as little as possible.

(2), (3), (4), and (7) describe methods to solve the routing problem for a virtual link and (1), (5), (6), (7), and (8) explain flow assignment in the virtual link. (1) and (5) can prevent from out of ordering packets and in (2) and (4), two constraints of max hop count and the maximum number of LSPs are considered when LSPs are set up.

Assume 100 flows which consist of 20 flows with Expedited Forwarding (EF) PHB and 80 flows with Assured Forwarding (AF) PHB in one link. In standard DiffServ, 20 EF flows can be given priority in admission for QoS and then $80 \mathrm{AF}$ flows can be assured. Since a virtual link can be made up of several LSPs, however, service differentiation in some LSPs would be degraded. For example, if the flows forwarded to $10 \mathrm{LSPs}, \mathrm{LSP} 1$ for $10 \mathrm{EF}$ flows and 1 AF flows, LSP2 for 2 EF flows and 2 AF flows and LSP3 LSP10 for 1 EF flow and 11 AF flow, in LSP1, a AF flow will be transported after forwarding $10 \mathrm{EF}$ flows, while AF flows will be transported after 1 EF traffic in LSP3 LSP10. In addition, the service differentiation in LSP2 including 2 AF flows and 2 EF flows will show different characteristics against that in LSP3 LSP10 including 1 EF flow and 11 AF flows.

In unfortunate case, only one service class is supported in one LSP of a virtual link. It can cause increasing labels. If there is a LSP of a virtual link for 1Mbps traffic with EF PHB and another LSP of the virtual link for $1 \mathrm{Mbps}$ traffic with AF $\mathrm{PHB}, \mathrm{EF}$ traffic and AF traffic would do not experience service differentiation because they are independently treated guaranteeing bandwidth in different path. This problem should be alleviated by handling the incoming flows.

To solve these problems, LSPs of the virtual link must be managed dynamically according to network status and the traffic in the virtual link is distributed among LSPs by the flow. The LSPs of the virtual link should have the similar traffic distribution to service classes to reduce service unbalancing.

\subsection{Algorithm}

We propose an algorithm to set up a virtual link and to manage the traffic of the virtual link. The Figure 4 shows the flow chart of the algorithm and follows illustrate the chart.

(1) A flow request including required bandwidth, service class and destination arrives.

(2) Determine all paths set to the destination

(3) Find a path and distribute the traffic in the virtual link until the flow is 
accepted. We propose threshold count, $\mathrm{K}$. If the number of path in the path set to egress node is more than threshold count, $\mathrm{K}$, processing delay for would degrade network performance.

(4) In the path set, select a maximum bandwidth path. Add the path to LSP set, the virtual link, and repeat

(5) If the traffic can be distributed in the virtual link, set up the LSP and allocate the distributed traffic. Or, include one more path in the virtual link until threshold count, $\mathrm{K}$ and repeat (3)

(6) If the flow can't be accepted, the flow will be blocked.

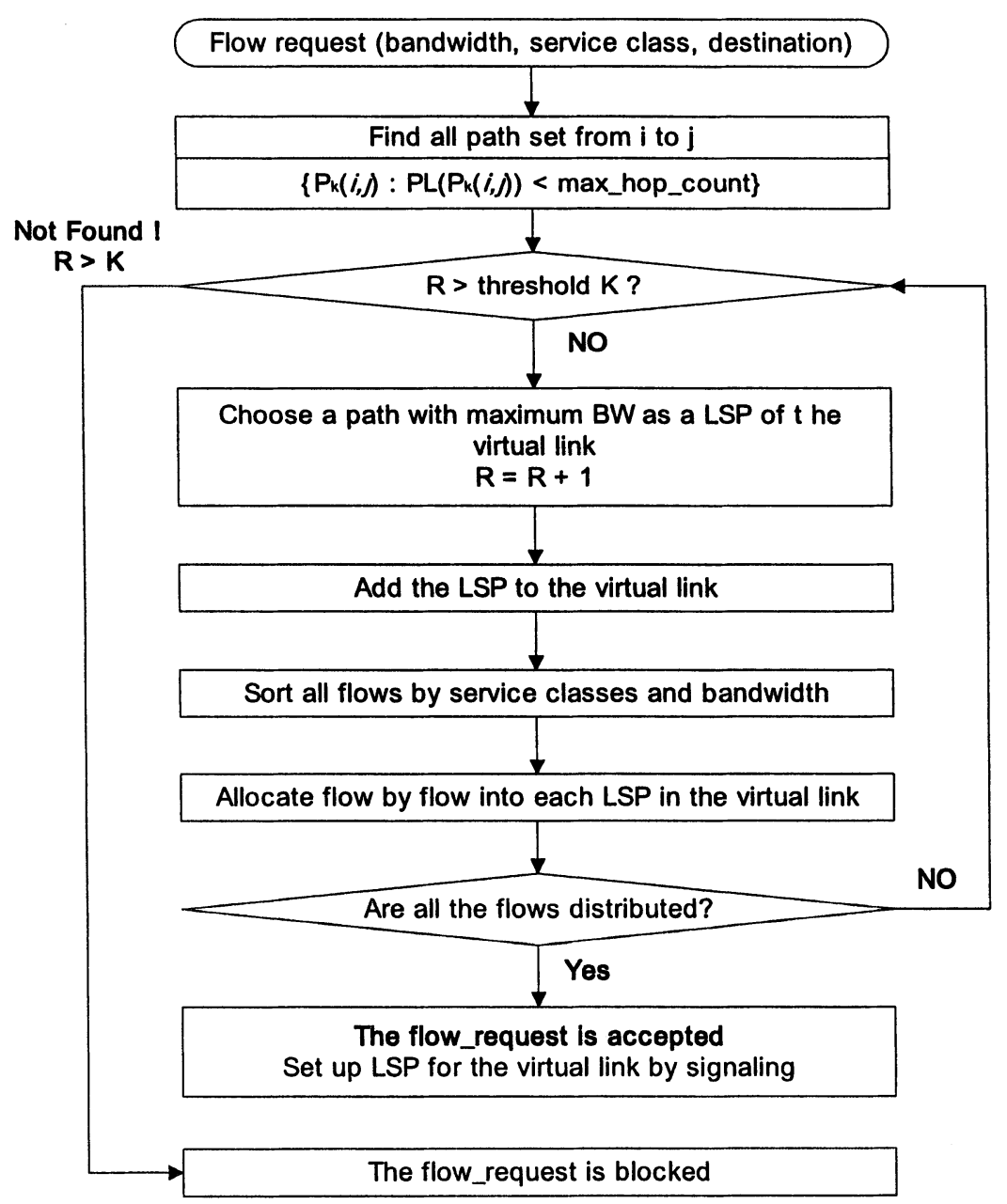

Figure 4. Algorithm of Flow Admission Control 


\section{COMPARISON}

This paper focuses the scheme to simply support QoS in MPLS network as compared to conventional IETF scheme. This section describes strong points and weak points through comparison between the IETF scheme and the proposed scheme. First, the network architecture of the IETF scheme is an integrated model of DiffServ and MPLS. In this scheme, PHB should be applied in every node to support QoS. Thus, it would be difficult to build up DiffServ over MPLS. However, it is easy and simple in proposed scheme to deploy DiffServ in MPLS network because only boundary edge router needs to be modified and PHB is applied in ingress LER using virtual link. In addition, the conventional scheme uses E-LSP or L-LSP, which restricts the number of services or increases the number of labels. But, the proposed scheme doesn't need any label encoding scheme.

Second, the IETF scheme can meet demanded service quality as exactly as DiffServ. In the proposed scheme, there can be several LSPs in the virtual link. The AF traffic in the LSPs of the virtual link could experience higher priority in quality of service than that in the IETF scheme. On the other hand, all EF traffic does not experience more priority than AF traffic in the proposed scheme because core LSRs classify arrival traffic only according to the LSP. Thus, the degree of the service differentiation in a LSP may be different from that of other LSPs included in common virtual link in proposed scheme..

Third, as centralized bandwidth broker may cause more processing delay in backbone network, we present QoS agent to distribute bandwidth broker's load. Virtual Link with guaranteed bandwidth can be set up by existing CR-LDP or RSVP-TE. Table 1 summarizes these comparisons between IETF scheme and proposed scheme.

Table 1. Comparison of two QoS Schemes

\begin{tabular}{|c|l|l|}
\hline & Existing scheme by IETF & \multicolumn{1}{c|}{ Proposed scheme } \\
\hline \multirow{5}{*}{ Deployment } & - Integrated model. & - Implements PHB at only \\
& - Difficult to support \\
& DiffServ over MPLS \\
& network. & $\begin{array}{l}\text { - Needs to extend only ingress } \\
\text { LER. }\end{array}$ \\
& & $\begin{array}{l}\text { - Easy to deploy by modifying } \\
\text { only edge nodes }\end{array}$ \\
\hline \multirow{5}{*}{ QoS guarantee } & - May not experience accurate \\
& & QoS in the virtual link. Thus to \\
& & improve service differentiation, \\
& distribute the traffic among the \\
& the same level as & LSPs by a flow. \\
& standard DiffServ. & - EF traffic can't be given strict \\
& & priority in the virtual link. \\
& & - AF traffic can be treated prior \\
& & to other EF traffic of other \\
& & LSPs in the virtual link. \\
\hline Label & - E-LSP : maximum 8 & - No need. \\
\hline
\end{tabular}




\begin{tabular}{|c|l|l|}
\hline $\begin{array}{c}\text { encoding } \\
\text { scheme for } \\
\text { DiffServ }\end{array}$ & $\begin{array}{c}\text { services. } \\
\text { - L-LSP : increasing } \\
\text { labels. }\end{array}$ & \\
\hline $\begin{array}{c}\text { Scheduler } \\
\text { complexity }\end{array}$ & $\begin{array}{l}\text { - LER : performs multiple } \\
\text { service. } \\
\text { - LSR : the same above as } \\
\text { LER. }\end{array}$ & $\begin{array}{c}\text { - LER : multiple service level } \\
\text { scheduling. } \\
\text { - LSR : single service level } \\
\text { scheduling. } \\
\text { Thus, simple operation. }\end{array}$ \\
\hline $\begin{array}{c}\text { Bandwidth } \\
\text { Broker }\end{array}$ & - Centralized System. & $\begin{array}{l}\text { - Distributed System } \\
\text { - Use QoS agent. }\end{array}$ \\
\hline \multicolumn{2}{|c}{} \\
\hline
\end{tabular}

\section{PERFORMANCE EVALUATION}

To evaluate the performance of the proposed scheme, in this section, two experiments are carried out. The first experimental topology is shown in Figure 5. In this model, a virtual link from an ingress LER to an egress LER is made up of a single LSP. Link bandwidth is $10 \mathrm{Mbps}$ and $80 \%$ of the link is reserved. A fixed packet size of 1000 bytes is used. We focus on the packet delay of foreground traffic (S1, S2, S3) in the virtual link. The background traffic (S4, S5, S6, S7, S8, S9) is inputted to model the competition in bottle neck. The reserved bandwidths of $\mathrm{S} 1, \mathrm{~S} 2, \mathrm{~S} 3$ are $0.32 \mathrm{Mbps}, 0.8 \mathrm{Mbps}$, and $0.72 \mathrm{Mbps}$ respectively. We generated $\mathrm{BE}$ class traffic more than the rate we configure, $1.92 \mathrm{Mbps}$, because $\mathrm{BE}$ traffic is not guaranteed in resource of LSPs. In this experiment, we observe the packet delay of the foreground traffic in several cases where background traffic is changed. In our simulations, loss rate is not considered since packet loss is not only closely related to a various queue size but also proportional to packet delay.

Figures 6 shows the simulation results when $\mathrm{BE}$ background traffic is changed from $1 \mathrm{Mbps}$ to $8 \mathrm{Mbps}$, and $\mathrm{AF}$ and $\mathrm{EF}$ traffic are fixed at $1.28 \mathrm{Mbps}$ and $3.2 \mathrm{Mbps}$ respectively. Figures 7 shows the simulation results when AF background traffic is changed from $1.5 \mathrm{Mbps}$ to $4 \mathrm{Mbps}$, and $\mathrm{EF}$ and $\mathrm{BE}$ traffic are fixed at $1.28 \mathrm{Mbps}$ and $2.88 \mathrm{Mbps}$ respectively. Figures 8 shows the simulation results when EF

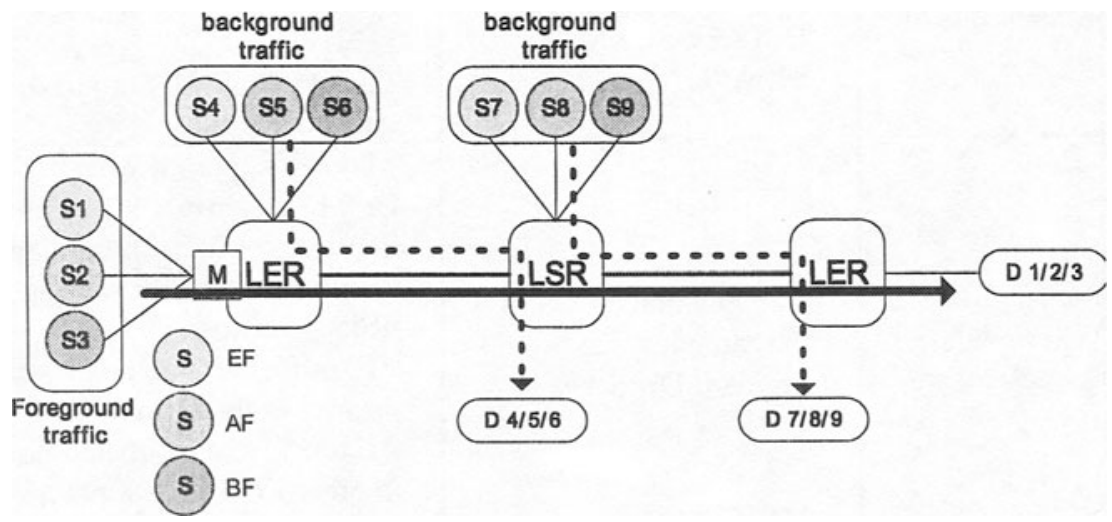

Figure 5. A network topology to evaluate the virtual link model in case of single path 
background traffic is changed from $0.1 \mathrm{Mbps}$ to $2.3 \mathrm{Mbps}$, and $\mathrm{AF}$ and $\mathrm{BE}$ traffic are fixed at $3.2 \mathrm{Mbps}$ and $2.88 \mathrm{Mbps}$ respectively. We see in the Figures while EF packet delay in IETF scheme is always very low due to the strict high priority in every node regardless of each class load of background traffic, EF packet delay in the proposed scheme is increasing in proportion to the background traffic load. This is because ingress node arranges packet order of incoming flows by PHB module of the LSP, but core node just guarantees the LSP's bandwidth using single level scheduler regardless of service class of the flows in the proposed scheme. In heavy link congestion region, $\mathrm{BE}$ packet delay is very rapidly increasing in IETF scheme compared with that in the proposed scheme. Therefore, it is observed that the IETF scheme may cause critical packet delay or loss for AF or BE traffic in heavy congestion region and that the proposed scheme can reduce the delay for $\mathrm{AF}$ or BE instead of increasing the delay of EF in the LSP. The proposed scheme, however, still makes service differentiation within the virtual link regardless of network environments.

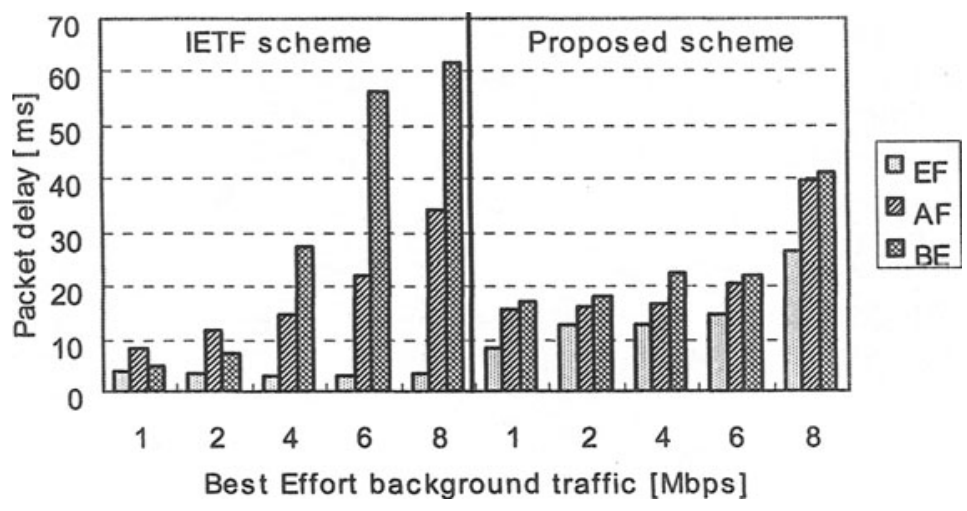

Figure 6. Foreground traffic packet delay according to BE background traffic

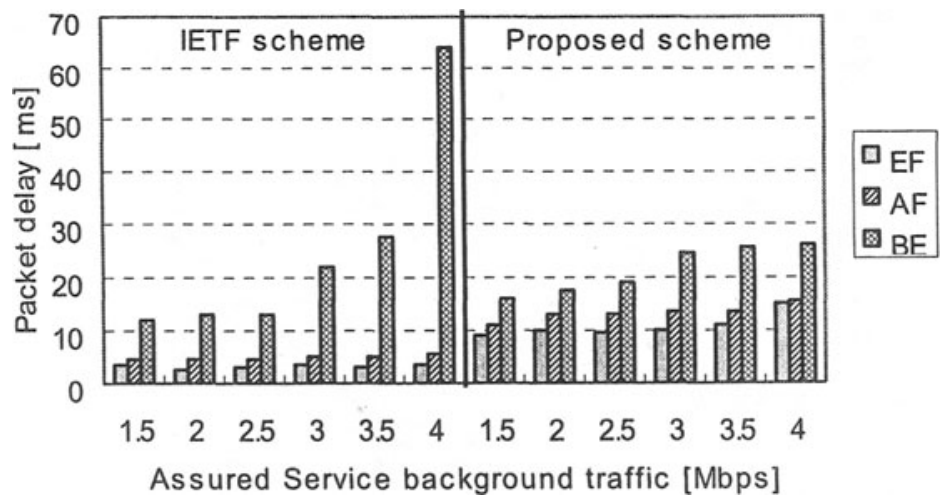

Figure 7. Foreground traffic packet delay according to AF background traffic 


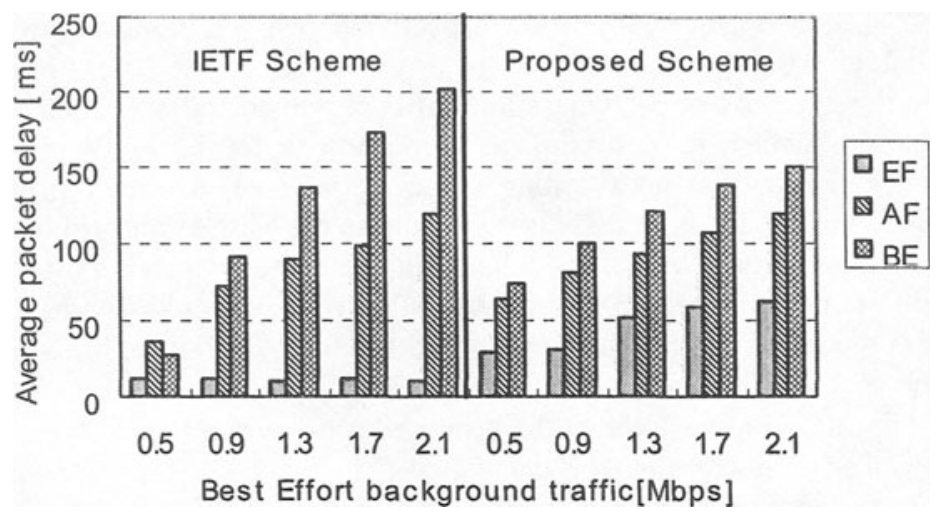

Figure10 . Foreground traffic average packet delay according to BE background traffic

In the next experiment, we observe the packet delay of foreground traffic which is forwarded through multiple LSPs included in a virtual link. Figure 9 shows the model. All links have 3Mbps capacities and LSP1, LSP2, and LSP3 has $0.267 \mathrm{Mbps}$, $0.533 \mathrm{Mbps}$, and $1.6 \mathrm{Mbps}$ capacities respectively. In this experiment, $\mathrm{BE}$ background traffic is varied, but AF and EF traffic are fixed. All bottle neck links reserve 2.4Mbps bandwidth for AF and EF traffic.

Figure 10 shows the average packet delay of foreground traffic through LSP1, LSP2, and LSP3 according to the BE background traffic. In heavy congestion cases of $1.7 \mathrm{Mbps}, 2.1 \mathrm{Mbps}$, AF traffic of the proposed scheme has the tendency of the

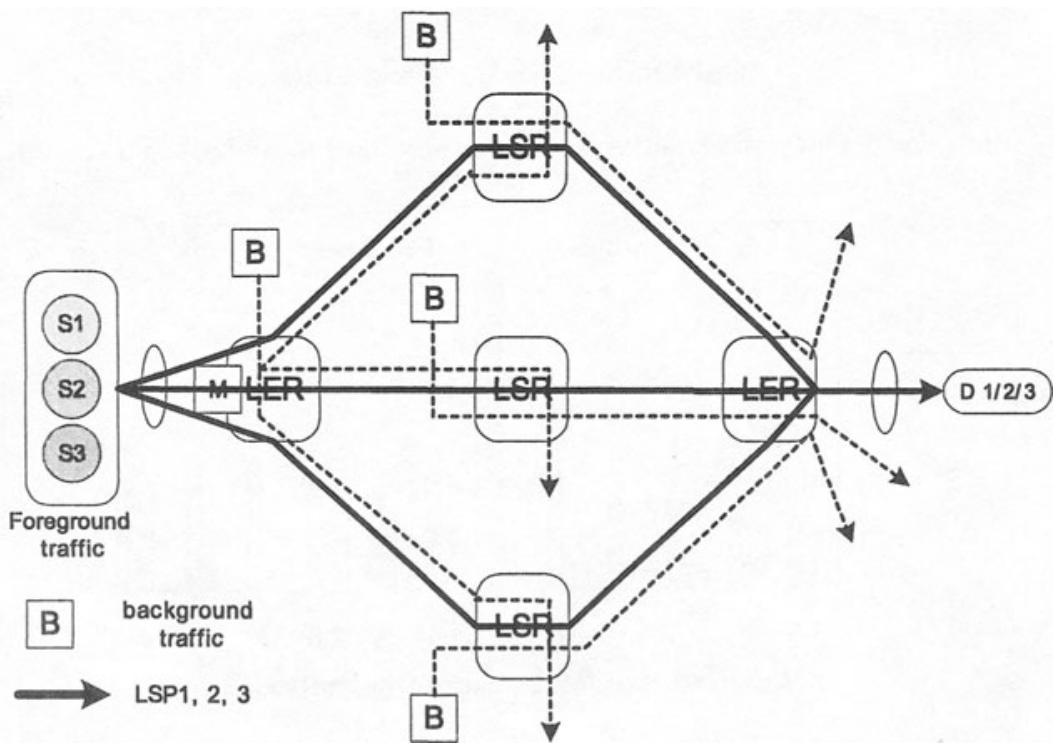

Figure 9. A network topology to evaluate the virtual link model in case of multiple path 
packet delay similar to that of IETF scheme. Also, the packet delay of BE in the proposed scheme is lower than that of IETF scheme, but EF traffic experiences more delay in the proposed scheme. From these simulation results, in the results, it is observed that the proposed scheme can provide QoS although the characteristics of service differentiation are obtuse in the case of multiple paths included in a virtual link as in the case of single path. Foreground traffic packet delay according to $\mathrm{BE}$ background traffic

\section{CONCLUSIONS}

The backbone network should be simple. This paper presents a simplified QoS scheme of MPLS based backbone network using virtual link. We first propose a concept of virtual link. The proposed scheme sets up a virtual link composed of a LSP set between an ingress LER and an egress LER and applies a PHB module to each LSP only at an ingress LER. And It doesn't use any label encoding scheme to identify the service class of packet. Existing CR-LDP or RSVP-TE is used to set up the virtual link without modification in this scheme. Thus, the proposed scheme needs to modify only MPLS edge routers to support QoS. Furthermore, a QoS agent for virtual management is proposed as a distributed system. In this scheme, there are two problems in virtual link management. One is what LSPs should be selected and the other is how all flows are assigned to the LSPs. We present a LSP management mechanism to solve these problems. When a flow request is arrived or an existing flow request is terminated, a set of LSPs is reconfigured and then all flows are redistributed among the set of LSPs by this mechanism. From our simulation results, we can conclude that the IETF scheme can meet demanded service quality as exactly as a standard DiffServ regardless of each class load of background traffic while EF packet delay in the proposed scheme is increasing in proportion to the background traffic load. The proposed scheme, however, can provide QoS although the characteristics of service differentiation are obtuse.

\section{REFERENCES}

[1] S. Blake, D. Blake, M. Carlson, E. Davies, Z. Wang, W. Weiss, "An Architecture for Differentiated Services," RFC2475, December 1998.

[2] Eric C. Rosen, A. Viswanathan, R. Callon, "Multiprotocol Label Switching Architecture," RFC 3031, January 2001.

[3] F. L. Faucheur, L. Wu, B. Davie, S. Dạvari, P. Vaananen, R. Krishnan, P. Cheval, J. Heinanen, "MPLS Support of Differentiated Service," Internet Draft<draftietf-mpls-diff-ext-09.txt>, April 2001.

[4] T. Nadeau, M. Tatham, T. Telkamp, J. Boyle, L. Fang, "Requirements for support of Diff-Serv-Aware MPLS Engineering" Internet Draft<draft-ietf-mpls-diff-tereqts-00.txt>, November 2000

[5] Melody Moh, Belle Wei and Jane Huijing Zhu, "Supporting Differentiated Services with Per-Class Traffic Engineering in MPLS," Computer Communications and Networks, January 2001, pp.354-360 
[6] O. Aboul-Magd, P. Ashwood-Smith, F. Hellstrand,K. Sundell, L. Andersson, R. Callon, R. Dantu, L. Wu, P. Doolan, T. Worster, N. Fredette, M. Girish,E. Gray, J. Halpern, J. Heinanen, T. Kilty, A. Malis, P. Vaananen "Constraint-Based LSP Setup using LDP," Internet Draft<draft-ieft-mpls-cr-ldp-06.txt>, November 2001 